\title{
Relacionando dieta e horários de captura entre duas espécies de morcegos frugívoros (Chiroptera, Phyllostomidae, Stenodermatinae)
}

\section{Relating Diet and Capture Time Between two Species of Frugivorous Bats (Chiroptera, Phyllostomidae, Stenodermatinae)}

\author{
Eduardo Ribeiro Sartore ${ }^{1}$; Nelio Roberto dos Reis ${ }^{2}$
}

\begin{abstract}
Resumo
Artibeus lituratus (Olfers, 1818) e Platyrrhinus lineatus (Geoffroy, 1810) são morcegos filostomídeos que frequentemente coexistem num mesmo local e consomem o mesmo alimento. Assim, o objetivo deste estudo foi obter dados sobre os hábitos alimentares das duas espécies, relacionando-os com seus horários de captura, na região onde se encontra o campus da Universidade Estadual de Londrina no norte do Paraná. As coletas foram realizadas entre Setembro de 2010 e Maio de 2011, com um esforço amostral de $12960 \mathrm{~m} 2 \mathrm{~h}$. A análise dos resultados contemplou o uso do Índice de Shannon, Índice de Pielou e Índice de Schoener. Capturaram-se 250 A. lituratus e 46 P. lineatus, e o primeiro consumiu frutos de dez espécies vegetais, enquanto o segundo utilizou apenas três espécies, ambos dando preferência aos gêneros Cecropia spp. e Ficus spp. A. lituratus apresentou o maior pico de captura 30 minutos após o pôr-do-sol e foi coletado durante todo o período. P. lineatus teve seu maior pico $01 \mathrm{~h}$ $45 \mathrm{~min}$ após o anoitecer e foi capturado em pequenos períodos. Com esses resultados, sugere-se que P. lineatus tem seu pico de forrageamento, no local de estudo, 75 minutos após A. lituratus, evitando competição direta. A. lituratus apresentou maior diversidade de alimentos, indicando maior potencial adaptativo para esta espécie em relação à outra.

Palavras-chave: Coexistência. Sobreposição de nichos. Diversidade alimentar. Área urbana.
\end{abstract}

\begin{abstract}
Artibeus lituratus (Olfers, 1818) and Platyrrhinus lineatus (Geoffroy, 1810) are phyllostomid bats that often coexist in the same local and consume the same feed. Therefore, the aim of this study was to obtain data about feeding habits of the two species, relating to their capture times, in the land where is the Universidade Estadual de Londrina, northern Paraná. The samples were attained among September 2010 and May 2011, with $12960 \mathrm{~m} 2 \mathrm{~h}$ of sampling effort. The results analysis contemplated the Shannon Index, Pielou Index and Schoener Index. 250 A. lituratus e 46 P. lineatus were captured, and while the first one consumed fruits from ten vegetable species, the second fed just three species, both preferring the Cecropia spp. and Ficus spp. genus. A. lituratus showed the higher capture peak 30 minutes after the sunset and was collected throughout the period. P. lineatus had its higher peak $01 \mathrm{~h} 45 \mathrm{~min}$ after nightfall and was captured in short periods. With these outcomes, is possible to imply that P. lineatus has a foraging peak, in the study place, 75 minutes after A. lituratus, avoiding straight competition. A. lituratus showed larger food diversity, indicating higher adaptative potential for this specie than for the other.
\end{abstract}

Key-words: Coexistence. Niche overlap. Food diversity. Urban area.

\footnotetext{
${ }^{1}$ Laboratório de Ecologia de Mamíferos, Centro de Ciências Biológicas, Universidade Estadual de Londrina E-mail: er.sartore@ bol.com.br. Autor para correspondência.

${ }^{2}$ Laboratório de Ecologia de Mamíferos, Centro de Ciências Biológicas, Universidade Estadual de Londrina. E-mail: nrreis@uel. br
} 


\section{Introdução}

AOrdem Chiroptera é muito diversa, constituindo a maioria das espécies de mamíferos em muitas áreas tropicais e subtropicais (TADDEI, 1983). Entre a Classe Mammalia, formam o grupo com a maior variedade de hábitos alimentares (REIS et al., 2007a), sendo a família Phyllostomidae a mais versátil na obtenção de recursos, podendo explorar frutos, néctar, pólen, folhas, insetos, vertebrados, sangue entre outros (PASSOS; GRACIOLLI, 2004).

Os morcegos frugívoros estão entre os vertebrados que mais contribuem com a dispersão de plantas, pois defecam em pleno voo promovendo uma "chuva de sementes" (LOBOVA; GEISELMAN; MORI, 2009), além de que um único indivíduo pode dispersar centenas delas por noite (FLEMING; SOSA, 1994). Essa eficiência de dispersão está associada com seu hábito de forrageio, sua mobilidade e com as grandes distâncias que percorrem em busca de alimentos (GALINDOGONZÁLEZ, 1998). Esse fato coopera para o estabelecimento de muitas espécies de plantas pioneiras, auxiliando os mecanismos de sucessão primária e regeneração florestal em áreas tropicais (CHARLES-DOMINIQUE, 1986; GORCHOV et al., 1993).

Na guilda alimentar dos frugívoros, encontramse duas espécies de quirópteros da família Phyllostomidae, Artibeus lituratus (Olfers, 1818) e Platyrrhinus lineatus (É. Geoffroy, 1810), com grande número de indivíduos na sua região de ocorrência (ZORTÉA, 2007) e, por isso, importantes alvos de estudo em várias áreas. Essas espécies semelhantes, do ponto de vista de guilda alimentar, coexistem em um mesmo lugar devido à heterogeneidade do ambiente e à maneira como elas utilizam as três dimensões básicas do nicho (espaço, tempo e alimento), geralmente com variação em pelo menos uma dessas três, evitando sobreposição (PIANKA, 1973). Segundo Humphrey e Bonaccorso (1979), os morcegos filostomídeos, endêmicos da região Neotropical são o grupo geralmente mais capturado e com maior diversidade encontrado nas comunidades de mamíferos. No entanto, sua predominância pode estar associada à metodologia de captura (CADEMARTORI et al., 2010), isto é, com redes-de-neblina em até $3 \mathrm{~m}$ de altura a nível de árvores frutíferas, método particularmente eficiente para a captura de espécies frugívoras (FINDLEY, 1993).

Artibeus lituratus é um morcego de grande porte, com antebraço podendo passar de $75 \mathrm{~mm}$ de comprimento (VIZOTTO; TADDEI, 1973) e massa corpórea atingindo até $90 \mathrm{~g}$ (observação pessoal). Pode habitar regiões florestadas, alteradas ou não, e áreas urbanas, sendo uma das espécies mais bem adaptada a estas últimas (BREDT; UIEDA, 1996). Sua dieta constitui-se basicamente de frutos, utilizando várias famílias vegetais, tais como Moraceae, Urticaceae, Solanaceae, Piperaceae entre outras (GARDNER, 1977), mas também pode se alimentar de insetos, recursos florais e ainda folhas (ZORTÉA; CHIARELLO, 1994; ZORTÉA; MENDES, 1993). Sua distribuição geográfica abrange desde o México até o norte da Argentina (SIMMONS, 2005).

Platyrrhinus lineatus apresenta tamanho médio, cujo antebraço varia de 43 a $50 \mathrm{~mm}$ de comprimento (VIZOTTO; TADDEI, 1973) e massa corpórea de 23 g para machos e 26,5 g para fêmeas (WILLIG, 1983). Pode ser encontrado tanto em ambientes úmidos, a exemplo da Floresta Atlântica em sua totalidade, quanto em ambientes mais xeromórficos, como a Caatinga e o Cerrado (ZORTÉA, 2007). Além disso, Bredt e Uieda (1996) mencionam que esta espécie encontra abundância de alimentos e abrigo em áreas urbanas. Alimenta-se predominantemente de frutos de Moraceae e Urticaceae e é endêmico à América do Sul, ocorrendo desde a Colômbia até a Argentina (WILLIG; HOLLANDER, 1987).

Frutos dos gêneros Cecropia Loefl. e Ficus L. são consumidos com frequência tanto por A. lituratus quanto por P. lineatus e podem ser coincidentes na alimentação destes num mesmo 
hábitat (HANDLEY; LEIGH, 1991; WENDELN; RUNKLE; KALKO, 2000). Essa correlação na dieta, juntamente com informações sobre os padrões horários e sazonais de atividades, proporcionam o entendimento da ocupação do nicho e do estabelecimento e manutenção das comunidades nos ecossistemas (PIANKA, 1969; SCHOENER, 1974). Esses padrões de atividades devem ajustar-se às variações de alguns fatores, como: disponibilidade de alimento, possibilidade de serem predados (ESBÉRARD; BERGALLO, 2008; JONES; RYDELL, 1994; KUNZ; ANTHONY, 1996) e competição com espécies de hábitos alimentares similares (KUNZ; ANTHONY, 1996).

A área deste estudo e arredores tem sido local de coleta de dados em vários trabalhos (MULLER; REIS, 1992; REIS et al., 1993; REIS; PERACCHI; ONUKI, 1993; REIS; LIMA, 1994; SEKIAMA, 1996), entretanto é necessário melhor conhecimento sobre essas espécies que estão habitando ambientes urbanos, e se estão partilhando os mesmos recursos, mais limitados nestas áreas se comparados a ambientes naturais. Atualmente, a região é um ambiente degradado, com $2 \%$ a $4 \%$ de sua cobertura vegetal original (SOARES; MEDRI, 2002), porém já representou grande extensão na Mata Atlântica (TOREZAN, 2002). Para que se tenham resultados mais conclusivos, salienta-se a relevância de estudos sobre o tema, já que o bioma vem sendo referência na conservação de espécies e ecossistemas (CONSERVAÇÃO INTERNACIONAL DO BRASIL, 2000).

Teve-se como objetivo obter dados sobre os hábitos alimentares de Artibeus lituratus e Platyrrhinus lineatus, relacionando-os com seus horários de captura, na região onde se encontra o campus da Universidade Estadual de Londrina, na região norte do Paraná.

\section{Material e Métodos}

Área de estudo

O estudo foi realizado no campus da Universidade Estadual de Londrina, Paraná, Brasil (Figura 1). Nesse local, assim como em todo o norte do Estado, a vegetação originalmente dominante era a Floresta Estacional Semidecidual, um ecossistema associado ao domínio de Mata Atlântica (TOREZAN, 2002), o qual abriga diversas espécies de morcegos de diferentes famílias, tais como Phyllostomidae, Molossidae, Vespertilionidae, Noctilionidae, Emballorunidae entre outras (REIS et al., 2007b).

O município está situado a $23^{\circ} 23^{\prime} 30^{\prime \prime} \mathrm{W}$ e $51^{\circ}$ 11' 5" S, apresenta uma altitude média de $600 \mathrm{~m}$, possui clima subtropical úmido, com temperatura média anual oscilando entre 22 e $25^{\circ} \mathrm{C}$. $\mathrm{O}$ índice pluviométrico anual da região está em torno de 1 $500 \mathrm{~mm}$, sendo os meses mais chuvosos dezembro, janeiro e fevereiro (com médias pluviométricas de 500 a $550 \mathrm{~mm}$ ) e os meses mais secos os de junho, julho e agosto, apresentando médias de 200, 250 e $300 \mathrm{~mm}$, respectivamente (REIS; PERACCHI; ONUKI, 1993).

O campus conta com área de 230 hectares e os locais utilizados nas coletas são constituídos em sua maior extensão por gramados e árvores esparsas, representadas, principalmente, por Aspidosperma polyneuron Müll.Arg. ("perobarosa", Apocynaceae), Ficus spp. ("figueiras", Moraceae), Caesalpinia peltophoroides Benth. ("sibipiruna", Fabaceae), Terminalia catappa L. ("sete-copas", Combretaceae), Spathodea nilotica Seem. ("bisnagueira”, Bignoniaceae) e diversas palmeiras (Arecaceae). Também se encontram algumas árvores frutíferas, destacando-se Morus spp. ("amoreiras", Moraceae), Psidium guayaba L. ("goiabeira", Myrtaceae), Artocarpus altilis (Parkinson) Fosberg ("fruta-pão", Moraceae), 
Persea americana Mill. (“abacateiro”, Lauraceae), assim como arbustos, dos quais se destacam Rhododendron sp. ("azaléia", Ericaceae), Bougainvillea sp. ("primavera", Nyctaginaceae) e Hibiscus sp. ("hibisco", Malvaceae) (REIS;
PERACCHI; ONUKI, 1993). Entre essas espécies vegetais há tanto nativas quanto exóticas. Existe também uma região florestada com 10 ha de mata nativa, todavia com ambiente alterado.

Figura 1 - Localização dos pontos de amostragem no campus da UEL no município de Londrina, Estado do Paraná.

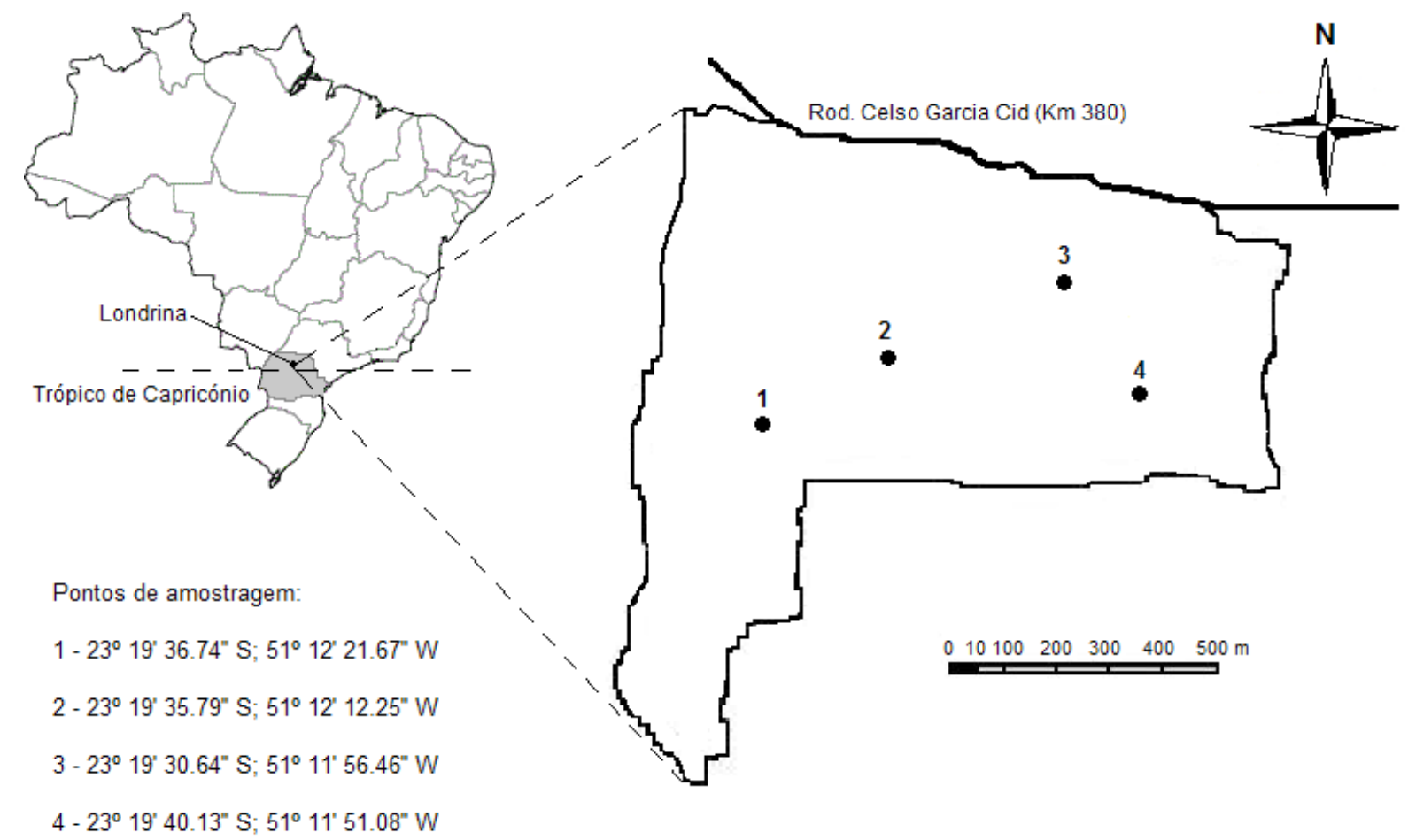

Fonte: Autores.

\section{Metodologia de Amostragem}

As capturas foram realizadas entre os meses de Setembro de 2010 a Maio de 2011, totalizando nove meses de coleta, sendo quatro noites em cada mês, onde se obteve um esforço amostral de $12960 \mathrm{~m}^{2} \mathrm{~h}$, calculado de acordo com Straube e Bianconi (2002). Foram selecionados quatro locais diferentes dentro do campus, destacando que a menor distância entre estes pontos foi de $240 \mathrm{~m} \mathrm{e}$ a maior, de $950 \mathrm{~m}$. Quatro redes-de-neblina (mistnets) foram usadas, com $9 \times 2,5 \mathrm{~m}$ de comprimento cada uma, abertas próximas a plantas dos gêneros Ficus e Cecropia principalmente, logo após o pôrdo-sol, e permaneceram assim durante quatro horas, sendo vistoriadas a cada $15 \mathrm{~min}$.

Com relação à retirada dos animais que são capturados nas redes, fez-se uso de luvas de couro para proteção contra mordedura, já que esses, como outros mamíferos, podem ser transmissores do vírus da raiva (SODRE; GAMA; ALMEIDA, 2010). Posteriormente foram identificados com uso de chaves dicotômicas (REIS et al., 1993; 
VIZOTTO; TADDEI, 1973) e anilhados no local, evitando repetição de dados por recaptura, e então acondicionados em sacos de algodão por aproximadamente 40 minutos antes de libertados. Como o trânsito de alimento através do sistema digestivo dos morcegos frugívoros é extremamente rápido (tipicamente 15-35 minutos (MORRISON, 1980)), esse tempo é suficiente para se obter sementes das fezes dos animais (DINERSTEIN, 1986). Também foram registrados os horários de captura dos espécimes.

A identificação das sementes, coletadas nas fezes dos animais, foi feita com o auxílio de um microscópio estereoscópico, comparando-se este material com sementes de plantas amostradas na área de estudo, previamente coletadas e identificadas, e por comparação do material do herbário da Universidade Estadual de Londrina (FUEL). Fezes contendo apenas polpa de frutos, como Terminalia catappa L. e Syagrus romanzoffiana (Cham.) Glassman, as quais possuem sementes grandes para serem ingeridas por morcegos, eram passíveis de identificação através da análise de cor, textura e cheiro, comprovando com a observação direta do forrageio dos animais.

\section{Análise Estatística}

Analisaram-se as amostras utilizando o Índice de Shannon (1948) como medida de amplitude de nicho trófico, que é dado por: $H^{\prime}=-\Sigma\left(p_{i} \ln p_{i}\right)$, onde pi é a proporção de um item alimentar pelo total de itens consumido pela espécie. A equabilidade das visitas foi calculada pelo Índice de Pielou, por meio da fórmula: J' = H'/H'max, sendo que H' $\max =$ $\operatorname{lnS}$, onde $\mathrm{S}$ é o número de recursos utilizados pela espécie (LUDWIG; REYNOLDS, 1988).
Os padrões de sobreposição alimentar das duas espécies foram avaliados de acordo com o Índice de Schoener (1970), que é dado pela fórmula: $\alpha$ $=1-0,5\left(\Sigma\left|p_{x i}-p_{y i}\right|\right)$, em que: $\alpha=$ sobreposição alimentar; $p_{x i}=$ proporção do item alimentar $\mathrm{i}$ na dieta da espécie $\mathrm{x} ; \mathrm{p}_{\mathrm{yi}}=$ proporção do item alimentar i na dieta da espécie y. Esse índice varia de 0 a 1, e a sobreposição é considerada biologicamente significativa quando o valor for igual ou superior a 0,6 (ZARET; RAND, 1971; WALLACE, 1981).

\section{Resultados}

Foi coletado um total de 336 indivíduos, sendo 250 da espécie Artibeus lituratus, 46 da espécie Platyrrhinus lineatus e os demais compreendem as espécies Eptesicus furinalis (d'Orbigny, 1847); Molossops neglectus Williams \& Genoways, 1980; Myotis nigricans (Schinz, 1821); Phyllostomus hastatus (Pallas, 1767) e Sturnira lilium (É. Geoffroy, 1810). A. lituratus consumiu Ficus citrifolia Mill., Ficus eximia Schott, Cecropia glaziovii Snethl., Cecropia pachystachya Trécul, Terminalia catappa e Syagrus romanzoffiana e de forma minoritária Ficus adhatodifolia Schott ex Spreng., Solanum paniculatum L., Piper aduncum L. e uma polpa de fruto não identificada. Enquanto P. lineatus consumiu apenas F. citrifolia, C. glaziovii e C. pachystachya (Figura 2).

Considerando os horários de captura, A. lituratus foi coletado em todos os horários em que as redes permaneceram abertas, com o maior pico aos 30 minutos após o pôr-do-sol, enquanto P. lineatus mostrou seu pico $01 \mathrm{~h} 15 \mathrm{~min}$ após este último (Figura 3). 
Figura 2 - Porcentagem de itens vegetais contidos nas fezes de morcegos coletados entre Setembro/2010 e Maio/2011 na Universidade Estadual de Londrina.

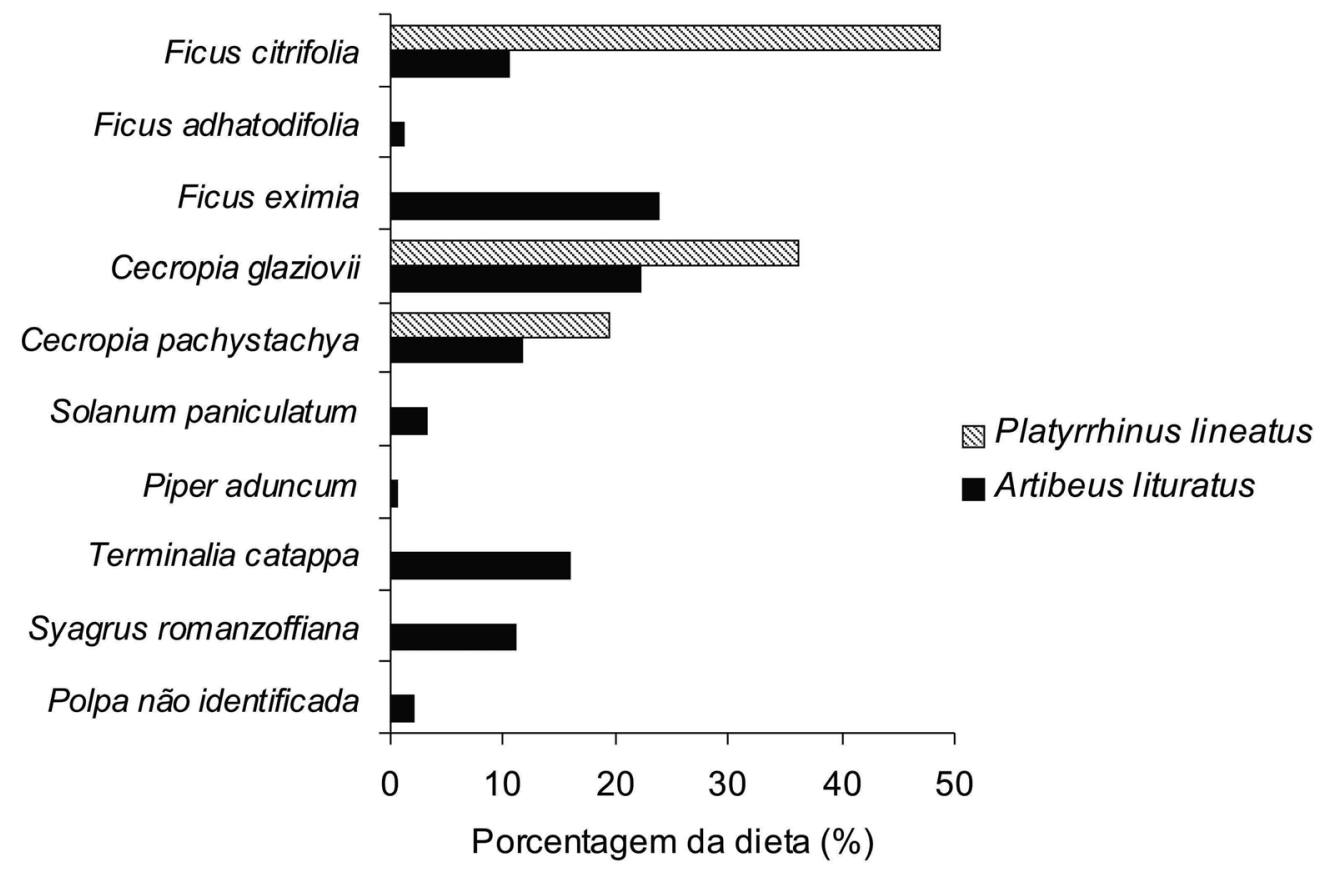

Fonte: Autores.

Figura 3 - Número de indivíduos e horário de captura após o anoitecer, de Artibeus lituratus e Platyrrhinus lineatus, no campus da Universidade Estadual de Londrina, no período entre Setembro/2010

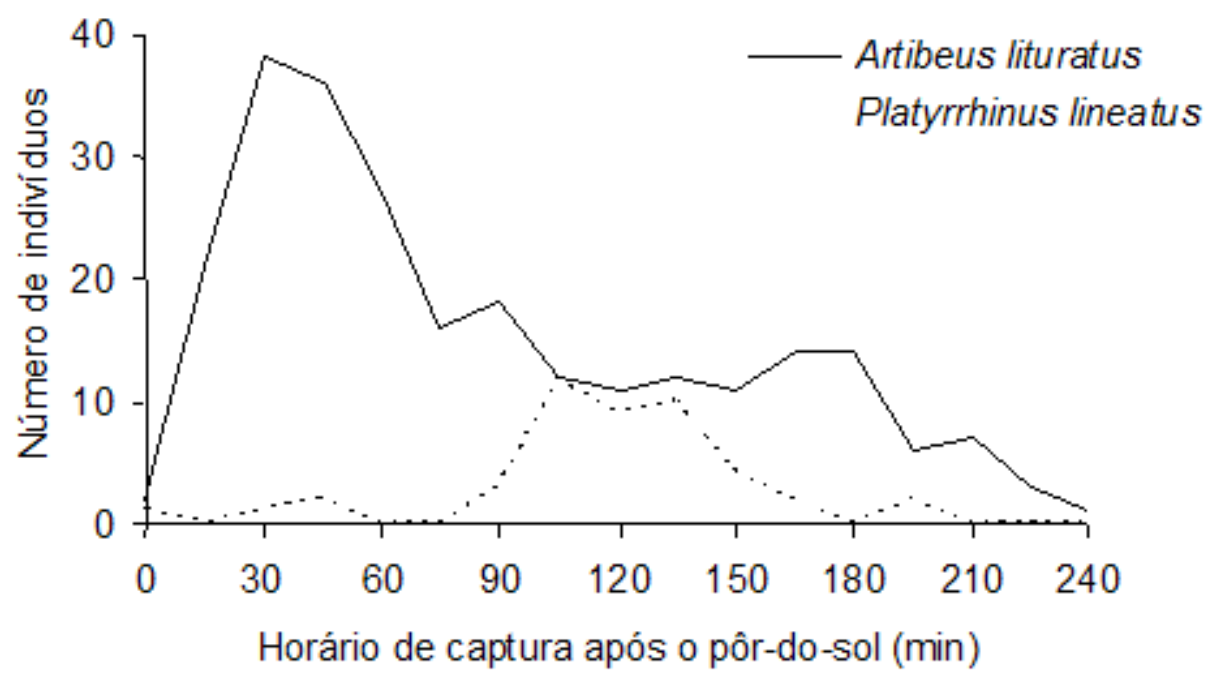

Fonte: Autores. 


\section{Discussão}

Frutos de Cecropia são considerados um dos principais itens alimentares de Artibeus lituratus e Platyrrhinus lineatus (SATO; PASSOS; NOGUEIRA, 2008), além de ter sido relatado como parte de sua dieta em diversas localidades do Brasil (GALETTI; MORELLATO, 1994; GARCIA; REZENDE; AGUIAR, 2000; PASSOS; GRACIOLLI, 2004), incluindo no Estado do Paraná (MULLER; REIS, 1992). Espécies de Moraceae, principalmente as do gênero Ficus, tem como Artibeus spp. e Platyrrhinus lineatus como seus principais dispersores de sementes em diversas localidades (DUMONT, 2003; GIANNINI; KALKO, 2004; GONÇALVES, 2009). Assim, explica-se a maior frequência de sementes de Ficus e Cecropia nas fezes de A. lituratus, e sua totalidade em P. lineatus. Com isso, torna-se claro o potencial de dispersão de sementes dessas plantas por esses morcegos.

Este padrão de dieta, já relatado por Fleming (1986), sugere preferência das duas espécies pelos dois gêneros de plantas. E ainda, a porcentagem total de amostras para os dois gêneros foi semelhante em ambos os morcegos. Isto possivelmente porque, para valores nutricionais, seus frutos podem ser complementares, já que os de Cecropia apresentam altas taxas de proteínas, carboidratos e lipídios (SILVA; PEREIRA FILHO; OLIVEIRA-PEREIRA, 2003), enquanto de Ficus são mais pobres nestes recursos e ricos em minerais, principalmente íons de cálcio e magnésio (FRANCENER, 2006). Sendo assim, acredita-se que uma das causas dessas espécies de morcegos buscarem esses itens alimentares com mesmo esforço, pode ser devido as suas propriedades nutricionais, propondo que isto foi estabelecido ao longo do curso evolutivo.

Apesar da preferência observada, Artibeus lituratus apresentou dieta diversificada, composta por frutos de dez espécies. Seu grande porte, que oferece musculatura mais desenvolvida melhorando a capacidade de voo, além de maior agressividade perante outro animal, e a habilidade de forragear durante vários períodos da noite, lhe confere grande sucesso em obter alimento (ZORTÉA, 2007), podendo ser mais limitado para P. lineatus, pelo seu menor tamanho, quando comparado a outra espécie. Neste trabalho, encontrou-se dominância de A. lituratus $(84,46 \%)$ sobre $P$. lineatus no local estudado, que pode ser interpretado através da diferença entre o número total de indivíduos capturados de cada espécie nesse local, refletindo na diversidade de itens alimentares encontrados em suas fezes e amplitude do horário de captura, ambos maiores para A. lituratus.

Taxas mais elevadas de consumo de Terminalia catappa e Syagrus romanzoffiana, abundantes no campus, por A. lituratus, podem ser explicadas pelo fato de que as espécies mais comuns em sua alimentação nem sempre estão em plena frutificação no local de estudo, ou seja, com número limitado de indivíduos frutificando. Além disso, essas espécies apresentam frutos maiores que as demais, incomuns na dieta de morcegos, o que impossibilitaria $P$. lineatus de carregá-los. Dessa forma, devido ao seu alto potencial adaptativo (REIS et al., 2000), A. lituratus procura frutos que possam estar mais disponíveis no ambiente, forrageando em uma mesma planta e, assim, gastando menor quantidade de energia, resultando em uma maneira de se conseguir muito alimento com o menor esforço possível.

Táticas ou componentes básicos de estratégias de forrageio incluem: amplitude da dieta, tamanho do grupo forrageador e atribuição do tempo de atividade entre diferentes áreas de alimentação (FLEMING, 1982). Desse modo, o maior número de capturas de A. lituratus em um mesmo horário que foram capturados P. lineatus, implica que o primeiro possa ter maior abundância de grupo de forrageio. Além disso, o valor de amplitude de nicho apresentase maior para A. lituratus $\left(\mathrm{H}^{\prime}=1,94\right)$, indicando que este seria mais generalista consumindo várias espécies, mas com preferência por certos recursos, indicada pela baixa equitabilidade na utilização 
destes $\left(\mathrm{J}^{\prime}=0,84\right)$. Platyrrhinus lineatus teve amplitude de nicho menor $\left(H^{\prime}=1,4\right)$, mostrando que este é mais especialista que o primeiro em ambiente urbanizado, onde os recursos alimentares são limitados a poucas espécies, sendo que algumas destas são de tamanho além da capacidade de consumo de P. lineatus, como Terminalia catappa ou mesmo Syagrus romanzoffiana, visto que consumiu poucos recursos, porém com grande uniformidade $\left(\mathrm{J}^{\prime}=1,28\right)$. Devido a essas espécies terem preferência pelos mesmos itens, já que Ficus spp. e Cecropia spp. representaram a maior parte da dieta de A. lituratus e a dieta por completo de P. lineatus, houve significativa sobreposição de nicho trófico $(\alpha=0,7)$.

Morcegos frugívoros parecem, de fato, comportarem-se como forrageadores oportunistas, utilizando seus principais itens alimentares do modo como são disponíveis no ambiente (HEITHAUS; FLEMING; OPLER, 1975; WILLIG; MOULTON, 1989). Dessa forma, outra explicação para a dieta encontrada para esses animais no local estudado é baseada na fenologia de frutificação, sendo que podem estar adaptados a modificar seu hábito alimentar de acordo com o ambiente em que estão vivendo (GAUTIER-HION et al., 1985), desde que encontrem as espécies vegetais consideradas de maior importância em sua dieta, como visto em outras localidades tropicais (BUMRUNGSRI; LEELAPAIBUL; RACEY, 2007; THIES; KALKO, 2004; THIES; KALKO; SCHNITZLER, 2006). Isso permite a esses morcegos habitarem áreas urbanas de modo semelhante a ambientes naturais, referente ao hábito alimentar.

Apesar de ocuparem o mesmo espaço e consumirem parcialmente o mesmo alimento, há coexistência entre as duas espécies, sugerindo que a competição é minimizada quando se refere ao forrageamento. Com isso, deve haver utilização de recursos em escala temporal diferente, para evitar sobreposição total de nicho entre os dois (PIANKA, 1973), visto que tiveram significativa sobreposição. No estudo, encontra-se diferença observada no horário de captura das duas espécies, inferindo que há alternância na ocupação do espaço através do horário para evitar competição direta, sendo que houve picos de captura diferentes para as duas espécies. Observa-se maior captura de P. lineatus 01h 45min após o pôr-do-sol, quando o número de A. lituratus capturados foi o mais baixo, indicando que antes desse horário a atividade deste último seja maior, representada pelo maior pico de captura logo após o anoitecer, e por isso é possível que a primeira espécie evite forragear neste horário no local estudado.

\section{Agradecimentos}

À Universidade Estadual de Londrina, à Prof ${ }^{\mathrm{a}}$ Dra. Silvia Helena Sofia pela revisão do manuscrito e à Bióloga Juliana Bombarda Ruim pelo auxílio nas coletas e apoio no desenvolvimento do trabalho.

\section{Referências}

BREDT, A.; UIEDA, W. Bats from urban and rural environments of the Distrito Federal, mid-western Brazil. Chiroptera Neotropical, Brasília, v. 2, n. 2, p. 54-57, 1996.

BUMRUNGSRI, S.; LEELAPAIBUL, W.; RACEY, P. A. Resource partitioning in sympatric Cynopterus bats in lowland tropical rain forest, Thailand. Biotropica, Washington, v. 39, n. 2, p. 241-248, Mar. 2007.

CADEMARTORI, C. V.; LIMA, C. S.; MARQUES, R. V.; MENDONÇA, C. M.; CABRAL, T. C.; PIRES, D. P. S.; DELAGNESE, D. G. Quiropterofauna registrada em um remanescente florestal do Domínio Mata Atlântica no Rio Grande do Sul, Brasil. Chiroptera Neotropical, v. 16, n. 1, ago. 2010.

CHARLES-DOMINIQUE, P. Inter-relations between frugivorous vertebrates and pioneer plants: Cecropia, birds and bats in French Guyana. In: ESTRADA, A.; FLEMING, T. H. (Ed.). Frugivores and seed dispersal. Dordrecht: Dr. W. Junk Publ., 1986. p. 119-135

CONSERVAÇÃO INTERNACIONAL DO BRASIL. Avaliação de ações prioritárias para conservação da biodiversidade da Mata Atlântica e Campos Sulinos. Brasília: MMA, 2000. 
DINERSTEIN, E. Reproductive ecology of fruit bats and the seasonality of fruit production in a Costa Rican Cloud Forest. Biotropica, Washington, v. 18, n. 4, p. 307-318, 1986.

DUMONT, E. R. Bats and fruits: an ecomorphological approach. In: KUNZ, T. H.; FENTON, M. Brock (Ed.). Bat ecology. Chicago: The Universe of Chicago Press, 2003. p. 398-429.

ESBÉRARD, C. E. L.; BERGALLO, H. G. Do bigger bats need more time to forage? Brazilian Journal of Biology, São Carlos, v. 68, n. 4, p. 631-637, nov. 2008.

FINDLEY, J. S. Bats: a community perspective. Cambridge: Cambridge University Press, 1993.

FLEMING, T. H. Foraging strategies of plant-visiting bats. In: KUNZ, T. H. (Ed.) Ecology of bats. New York: Spring Street, 1982. p. 287-325.

FLEMING, T. H. Opportunism versus specialization: evolution of feeding strategies in frugivorous bats. In: ESTRADA, A.; FLEMING, T. H. (Ed.). Frugivores and seed dispersal. Dordrecht: W. Junk Puplisher, 1986. p. 105-118.

FLEMING, T. H.; SOSA, V. J. Effects of nectarivorous and frugivorous mammals on reproductive success of plants. Journal of Mammalogy, Lawrence, v. 75, n. 4, p. 845-851, 1994.

FRANCENER, S. M. C. Análise nutricional dos frutos de Piper, Solanum e Ficus e sua importância na dieta dos morcegos. 2006. Dissertação (Mestrado em Química) Universidade Federal do Paraná, Curitiba, Paraná, Brasil.

GALETTI, M.; MORELLATO, L. P. C. Diet of the large fruit-eating bat Artibeus lituratus in a forest fragment in Brasil. Mammalia,Paris, v. 58, n. 4, p. 661-665, 1994.

GALINDO-GONZÁLEZ, J. Dispersión de semillas por murciélagos: su importância em la conservación y regeneración del bosque tropical. Acta Zoológica Mexicana, Ciudad del México, v. 73, p. 57-74, 1998.

GARCIA, Q. S.; REZENDE, J. L. P.; AGUIAR, L. M. S. Seed dispersal by bats in a disturbed area of Southeastern Brazil. Revista de Biologia Tropical, San José, v. 48, n. 1, p. 125-128, mar. 2000.

GARDNER, A. L. Feeding habits. In: BAKER, R. J.; JONES JUNIOR, J. K.; CARTER, D. C. (Ed.). Biology of the bats of the new world family phyllostomatidae. Lubbock: Texas Tech University, 1977.

GAUTIER-HION, A. J.; DUPLANTIER, R.; QURIS, F.; FEER, F.; SOURD, C.; DECOUX, J. P.; DUBOST, G.; EMMONS, L.; ERARD, C.; HECKETSWEILER, P.; MOUNGAZI, A.; ROUSSILHON, C.; THIOLLAY, J. M. Fruit characters as a basis of a fruit choice and seed dispersal in a tropical forest vertebrate community. Oecologia, New York, v. 65, n. 3, p. 324-337, 1985.

GIANNINI, N. P.; KALKO, E. K. V. Trophic structure in a large assemblage of Phyllostomid bats in Panama. Oikos, Lund, v. 105, p. 209-220, 2004.

GONÇALVES, F. Morcegos vetores de pólen e dispersores de sementes no Pantanal. 2010. $32 \mathrm{f}$. Dissertação (Mestrado em Ecologia) - Universidade Federal do Mato Grosso do Sul, Campo Grande, 2010.

GORCHOV, D. L.; CORNEJO, F.; ASCORRA, C.; JARAMILLO, M. The role of seed dispersal in the natural regeneration of rain forest after strip-cutting in the Peruvian Amazon. Vegetatio, n. 107, p. 339-349, 1993.

HANDLEY Jr., C. O.; LEIGH Jr., E. G. Diet and food supply. In: HANDLEY Jr., C. O.; WILSON, D. E.; GARDNER, A. L. (Ed.). Demography and natural history of the common fruit bat artibeus jamaicensis on barro Colorado Island, Panama. Washington: Smithsonian Institution Press, 1991. p. 137-140.

HEITHAUS, E.R.; FLEMING, T. H.; OPLER, P. A. Foraging patterns and resource utilization in seven species of bats in a seasonal tropical forest. Ecology, Washington, v. 56, p. 841-854, 1975.

HUMPHREY, S. R.; BONACCORSO, F. J. Biology of bats of the New World. Special Publications Museum, Texas, v. 16, p. 409-441, 1979.

JONES, G.; RYDELL, J. Foraging strategy and predation risk as factors influencing emergence time in echolocating bats. Philosophical Transactions: Biological Sciences, London, v. 346, p. 445-455, 1994.

KUNZ, T. H.; ANTHONY, E. L. P. Variation in nightly emergence behavior in the little brown bat, Myotis lucifugus (Chiroptera: Vespertilionidae). In: GENOWAYS, H. H.; BAKER, R. J. Contributions mammalogy: a memorial volume honoring J. Lubbock: Texas Tech University Press, 1996. p. 225-236.

LOBOVA, T. A.; GEISELMAN, C. K.; MORI, S. A. Seed dispersal by bats in the neotropics. Bronx, New York: The New York Botanical Garden, 2009.

LUDWIG, J. A.; REYNOLDS, J. F. Statistical ecology: a primer on methods and computing. New York: John Wiley \& Sons, 1988.

MORRISON, D. W. Efficiency of food utilization by fruit bats. Oecologia, New York, v. 45, p. 270-273, 1980.

MULLER, M. F.; REIS, N. R. Partição de recursos alimentares entre quatro espécies de morcegos frugívoros (Chiroptera, Phyllostomidae). Revista Brasileira de Zoologia, Curitiba, v. 9, n. 3-4, p. 345-355, 1992. 
PASSOS, F. C.; GRACIOLLI, G. Observações da dieta de Artibeus lituratus (Olfers) (Chiroptera, Phyllostomidae) em duas áreas do sul do Brasil. Revista Brasileira de Zoologia,Curitiba, v. 21, n. 3, p. 487-489, set. 2004.

PIANKA, E. R. The structure of lizards communities. Annual Review of Ecology and Systematics, Palo Alto, v. 4, p. 53-74, 1973.

PIANKA, E. R. Sympatry of desert lizards (Ctenotus) in western of Australia. Ecology, Washington, v. 50, p. 1012-1030, 1969.

REIS, N. R.; LIMA, I. P. Primeiro levantamento de morcegos em três grandes unidades de conservação na bacia do rio Tibagi. In: CONGRESSO DE ECOLOGIA DO BRASIL, 2., 1994, Londrina. Anais... Londrina: [s. n.], 1994.

REIS, N. R.; MUller, M. F.; SOARES, E. S.; PERACCHI, A. L. Lista e chave de quirópteros do Parque Estadual Mata dos Godoy e arredores, Londrina, Paraná. Semina: Ciências Biológicas/Saúde, Londrina, v. 14, n. 2 , p. 120-126, 1993.

REIS, N. R.; PERACCHI, A. L.; ONUKI, M. K. Quirópteros de Londrina, Paraná, Brasil (Mammalia, Chiroptera). Revista Brasileira de Zoologia, Curitiba, v. 10, n. 3, p. 371-381, 1993.

REIS, N. R.; PERACCHI, A. L.; PEDRO, W. A.; LIMA, I. P. Morcegos do Brasil. Londrina: Nelio Roberto dos Reis, 2007b.

REIS, N. R.; PERACCHI, A. L.; SEKIAMA, M. L.; LIMA, I. P. Diversidade de morcegos (Mammalia, Chiroptera) em fragmentos florestais no estado do Paraná, Brasil. Revista Brasileira de Zoologia, Curitiba, v. 17, n. 3, p. 697-704, 2000.

REIS, N. R.; SHIBATTA, O. A.; PERACCHI, A. L.; PEDRO, W.A.; LIMA, I. P. Sobre os morcegos brasileiros. In: REIS, N. R.; PERACCHI, A. L.; PEDRO, W. A.; LIMA, I. P. (Ed.). Morcegos do Brasil. Londrina: Nelio Roberto dos Reis, 2007a. p. 17-25.

SATO, T. M.; PASSOS, F. C.; NOGUEIRA, A. C. Frugivoria de morcegos (Mammalia, Chiroptera) em Cecropia pachystachya (Urticaceae) e seus efeitos na germinação das sementes. Papéis Avulsos de Zoologia, São Paulo, v. 48, n. 3, 2008.

SCHOENER, T. W. Non-synchronous spatial overlap of lizards in patchy habitats. Ecology, New York, v. 51, p. 408- 418, 1970.

SCHOENER, T. W. Resource partitioning in ecological communities. Science, v. 185, n. 4145, p. 27-39, 1974.

SEKIAMA, M. L. Estrutura da comunidade de quirópteros
(Chiroptera: Mammalia) no Parque Estadual Mata dos Godoy, Londrina, Paraná. 1996. Dissertação (Mestrado em Zoologia) - Universidade Federal do Paraná, Curitiba.

SHANNON, C. E. The mathematical theory of communication. In: SHANNON, C. E.; WEAVER, W. (Ed.), The mathematical theory of communication. Urbana: University Illinois Press, 1948. p. 3-91.

SILVA, J. A. M.; PEREIRA FILHO, M.; OLIVEIRAPEREIRA, M. I. Frutos e sementes consumidos pelo tambaqui, Colossoma macrompum (Cuvier, 1818) incorporados em rações: digestibilidade e velocidade de trânsito pelo trato gastrointestinal. Revista Brasileira de Zootecnia, Viçosa, v. 32, n. 6, dez. 2003.

SIMMONS, N. B. Order Chiroptera. In: WILSON, D. E.; REEDER, D. M. (Ed.). Mammal species of the world: a taxonomic and geographic reference. 3. ed. Baltimore: Johns Hopkins University Press, 2005. p. 312-529.

SOARES, F. S.; MEDRI, M. E. Alguns aspectos da colonização da bacia do rio Tibagi. In: MEDRI, M. E.; BIANCHINI, E.; SHIBATTA, O. A.; PIMENTA, J. A. (Ed.). A bacia do rio Tibagi. Londrina: Editora dos Editores, 2002. p. 69-79.

SODRE, M. M.; GAMA, A. R.; ALMEIDA, M. F. Updated list of bat species positive for rabies in Brazil. Revista do Instituto de Medicina Tropical de São Paulo, São Paulo, v. 52, n. 2, abr. 2010.

STRAUBE, F. C.; BIANCONI, G. V. Sobre a grandeza e a unidade utilizada para estimar esforço de captura com utilização de redes-de-neblina. Chiroptera Neotropical, Brasília, v. 8, n. 1-2, p. 150-152, 2002.

TADDEI, V. A. Morcegos: algumas considerações sistemáticas e biológicas. Brazilian Archives of Biology and Technology, Curitiba, v. 48, n. 2, p. 161-170, 1983.

THIES, W.; KALKO, E. K. V. Phenology of neotropical pepper plants (Piperaceae) and their association with their main dispersers, two short-tailed fruit bats, Carollia perspicillata and C. castanea (Phyllostomidae). Oikos, Lund, v. 104, n. 2, p. 362-376, 2004.

THIES, W.; KALKO, E. K. V.; SCHNITZLER, H. A. Influence of environment and resource availability on activity patterns of Carollia castanea (Phyllostomidae) in Panama. Journal of Mammalogy, Lawrence, v. 87, n. 2, p. 331-338, 2006.

TOREZAN, J. M. D. Nota sobre a vegetação da bacia do rio Tibagi,. In: MEDRI, M. E.; BIANCHINI, E.; SHIBATTA, O. A.; PIMENTA, J. A. (Ed.). A bacia do rio Tibagi. Londrina: Editora dos Editores, 2002. p. 103-107. 
VIZOTTO, L. D.; TADDEI, V. A. Chave para a determinação de quirópteros brasileiros. Revista da Faculdade de Filosofia Ciências e Letras São José do Rio Preto - Boletim de Ciências, São José do Rio Preto, n. 1, p. 1-72, 1973.

WALLACE, R. K. An assessment of the diet overlap indexes. Transactions of the American Fisheries Society, Bethesda, v. 110, p. 72-76, 1981.

WENDELN, M. C.; RUNKLE, J. R.; KALKO, E. K. V. Nutritional values of 14 fig species and bat feeding preferences in Panama. Biotropica, Washington, v. 32, n. 3, p. 489-501, 2000.

WILLIG, M. R. Composition, microgeographic variation, and sexual dimorphism in Caatingas and Cerrado bat communities from northeast Brazil. Bulletin of the Carnegie Museum of Natural History, Pittsburg, v. 23, p. 1-131, 1983.

WILliG, M. R.; HOLLANDER, R. R. Vampyrops lineatus. Mammalian Species, New York, n. 275, p. 1-4, 1987.

WILLIG, M. R.; MOULTON, M. P. The role of stochastic and deterministic processes in structuring Neotropical bat communities. Journal of Mammalogy, Lawrence, v. 70, n. 2, p. 323-329, 1989.

ZARET, T. M.; RAND, A. S.. Competition in tropical stream fishes: support for the competitive exclusion principle. Ecology, New York, v. 52, n. 2, p. 336-342, 1971.

ZORTÉA, M. Subfamília Stenodermatinae. In: REIS, N. R.; PERACCHI, A. L.; PEDRO, W. A.; LIMA, I. P. Morcegos do Brasil. Londrina: Nelio Roberto dos Reis, 2007. p. 107-128.

ZORTÉA, M.; CHIARELLO, A. G. Observations on the big fruit-eating bat, Artibeus lituratus in an urban reserve of south east Brazil. Mammalia, Paris, v. 58, n. 4, p. 665670, 1994.

ZORTÉA, M.; MENDES, S. L. Folivory in the big fruit-eating bat, Artibeus lituratus (Chiroptera, Phyllostomidae) in eastern Brazilian. Journal of Tropical Ecology, Cambridge, v. 9, p. 117-120, 1993.

Recebido em 21 de abril de 2011

Aceito em 20 de abril de 2012 
